\title{
UN ESTUDIO SOBRE EL PAPEL DE LAS ANALOGÍAS EN LA CONSTRUCCIÓN DEL MODELO CINÉTICO-MOLECULAR DE LA MATERIA
}

\author{
Oliva, J.M. ${ }^{1}$; Aragón, M.M. ${ }^{2}$; Bonat, M. ${ }^{3}$ y Mateo, J. ${ }^{4}$ \\ ${ }^{1}$ Centro de Profesorado de Cádiz \\ ${ }^{2}$ IES Drago. Cádiz \\ ${ }^{3}$ IES Mar de Cádiz. El Puerto de Santa María (Cádiz) \\ ${ }^{4}$ IES La Pedrera Blanca. Chiclana de la Frontera (Cádiz)
}

\begin{abstract}
Resumen. En este trabajo se propone, en primer lugar, un marco teórico para analizar el papel de las analogías en la construcción de modelos sobre fenómenos científicos por parte de los alumnos. Tomando como base dicho marco, se fundamenta, en segundo lugar, un diseño didáctico concreto encaminado a facilitar la construcción del modelo cinético-molecular de la materia a través de analogías, en alumnos de educación secundaria obligatoria. Finalmente, se evalúa el diseño didáctico empleado comparando los resultados finales alcanzados en pruebas de interpretación y predicción de fenómenos en un grupo experimental y en otro de control. Los resultados obtenidos parecen indicar que las analogías jugaron un papel relevante en la comprensión de los alumnos que fueron enseñados a través de dicho recurso. Éstos aportaron, finalmente, modelos explicativos más acordes con el modelo científico que los alumnos que fueron enseñados según un diseño semejante pero sin analogías, si bien las diferencias encontradas fueron pequeñas.

Palabras clave. Analogías, diseño didáctico, evolución cognitiva, modelos mentales, modelo cinético-molecular.
\end{abstract}

Summary. In order to analyze the role of the analogies on the model construction about scientific phenomena in the students, a theoretical framework is firstly proposed. From this framework, a concrete didactic design is also proposed with regard to facilitating the construction of a kinetic-molecular model of matter through analogies, in students of compulsory secondary education. Finally, the didactic design used is evaluated, comparing the final results in the interpretation and prediction of phenomena between an experimental group and a control one. The post-test results indicate that the analogies used played a relevant role in the learning process of the pupils who were taught through the mentioned resource. They provided, finally, explanatory models more in agreement with the scientific model than the pupils who were taught according to a similar design but without analogies, although the differences found were small.

Keywords. Analogies, cognitive development, didactic design, mental models, kinetic-molecular model.

\section{INTRODUCCIÓN}

Durante las dos últimas décadas se han realizado notables esfuerzos para esclarecer cuáles son las dificultades que tienen los alumnos en el aprendizaje de nociones relativas al modelo cinético-molecular. Muchas de esas dificultades parecen descansar sobre la existencia de concepciones implícitas que se adaptan mejor a sus experiencias cotidianas que los modelos científicos que se pretende enseñar. Concretamente, como bien ha sintetizado Benarroch (2000, 2001), estas dificultades consisten en: a) Una propensión a imaginar la materia en términos macroscópicos, en vez de hacerlo a partir de su carácter discontinuo o molecular.

b) Una tendencia a trasladar las propiedades macroscópicas de un sistema hacia las microscópicas.

c) Una resistencia a considerar los gases como sistemas materiales. 
d) La conceptualización, en el mejor de los casos, de un modelo corpúscular estático.

Con objeto de superar este tipo de dificultades, y de acuerdo con algunas interpretaciones que se han hecho de los modelos de cambio conceptual, muchos autores han sugerido el uso del conflicto conceptual con la experiencia como instrumento para desencadenar una «sustitución» de las ideas intuitivas por las ideas científicas que se pretenden enseñar. Sin embargo, por diversas razones, las propuestas didácticas concretas desarrolladas en este sentido no han funcionado todo lo bien que se esperaba (Dreyfus et al., 1990; Furió et al., 1994; Oliva, 1999). Por ello, parece lógico pensar en otras formas alternativas de conceptualizar el aprendizaje y otras maneras de dirigir la enseñanza en general y de estos temas en particular (Oliva, 1999).

En este sentido, no podemos olvidar el interés despertado últimamente por el aprendizaje a través de analogías, no sólo desde el ámbito de investigación en psicología (ver la revisión realizada por González-Labra, 1997), sino también desde de la didáctica de las ciencias (Duit, 1991; Dagher, 1995). Por este motivo, nos ha parecido interesante analizar el papel de las analogías en el aprendizaje de estos temas y en la superación de las concepciones alternativas, sobre todo teniendo en cuenta el escaso eco que las analogías parecen haber tenido en nuestro país como objeto de investigación (Aragón, Bonat, Oliva y Mateo, 1999).

Concretamente, la finalidad del estudio era comprobar la repercusión del uso de analogías en la asimilación del modelo corpuscular de la materia. Con tal fin diseñamos y aplicamos una unidad didáctica dedicada al estudio del modelo cinético-molecular y los estados de la materia, adoptando el uso de analogías como núcleo estructurante $\mathrm{y}$ vertebrador de los contenidos tratados. Se trataba de evaluar la propuesta, analizando los modelos explicativos finales utilizados por los alumnos al interpretar y predecir fenómenos físicos.

\section{MODELOS EN LOS ALUMNOS Y MODELOS EN LAS CIENCIAS}

Hoy día, el estudio de las analogías se encuentra muy cercano al ámbito de investigación sobre modelos (Oliva, Aragón, Mateo y Bonat, 2001). Por ello, en este trabajo la idea de modelo juega un papel central como unidad de conocimiento utilizado para comprender un fenómeno o resolver una situación dada.

Se utiliza con distintas acepciones y significados entre los que existen algunas conexiones importantes, pero también diferencias notables que conviene tener presente. A la hora de establecer diferenciaciones utilizaremos como marco teórico una adaptación del esquema de aprendizaje a través de modelos propuesto por Clement (2000). Según este autor, el aprendizaje consistiría en la evolución cognitiva a través de distintos modelos intermedios que tomarían como referente el modelo consensuado por los expertos o, lo que es lo mismo, el modelo aceptado por la comunidad científica. Esta continuidad entre las concepciones y modelos iniciales de los alumnos y el modelo objeto del aprendizaje sería posible, según Clement (2000), gracias a la existencia simultánea en los alumnos de modelos y concepciones iniciales útiles que pueden servir de anclaje de los nuevos modelos generados.

Sobre este supuesto básico hemos incorporado dos ideas adicionales, configurando así nuestra propia perspectiva de aprendizaje a través de modelos que se expone en la figura 1.

Figura 1

Adaptación del esquema de Clement (2000).

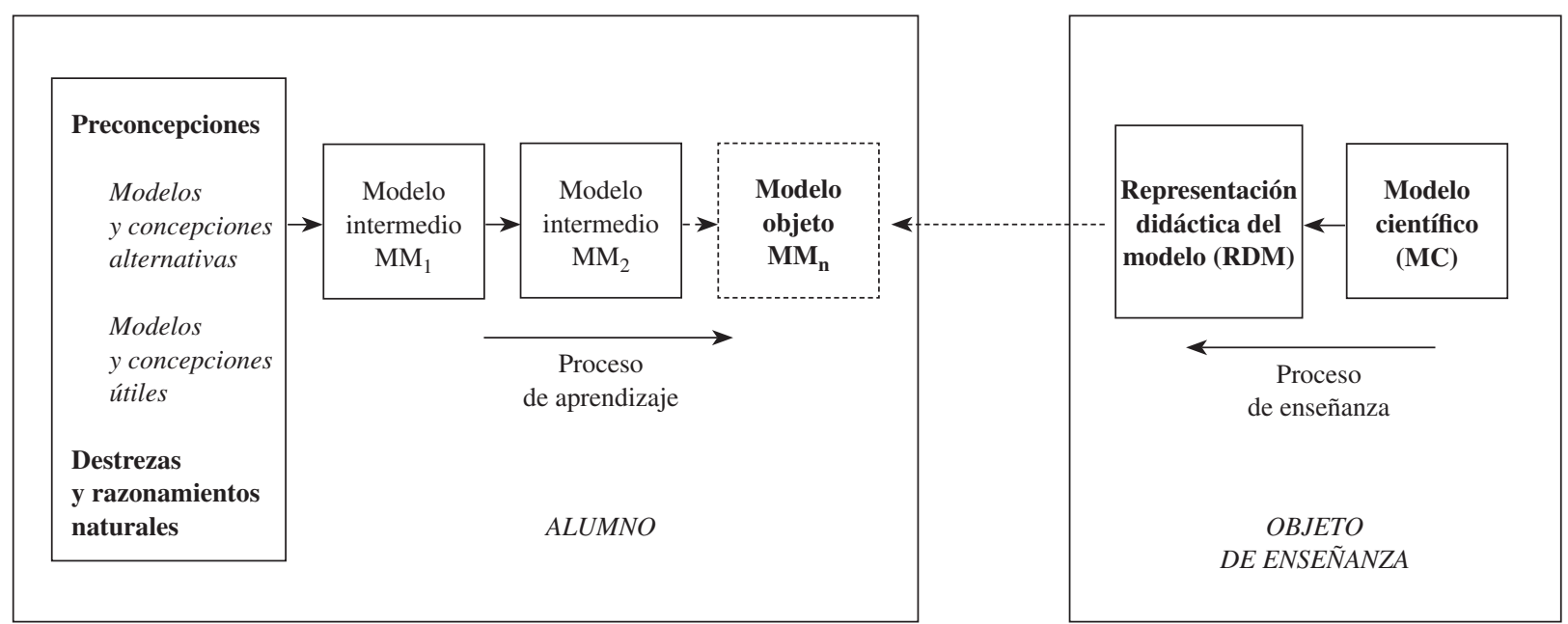


Nuestra primera aportación al esquema de Clement consiste en discernir entre conocimiento interno y externo al alumno (Marín, 2003) o, lo que es lo mismo, entre conocimiento del estudiante y conocimiento o contenido a enseñar. La segunda se refiere a la necesidad de diferenciar entre contenido científico y contenido escolar, considerando éste último como resultado de la transposición didáctica del primero y verdadero referente externo del aprendizaje.

Con vistas a tener en cuenta estas matizaciones, el esquema de la figura 1 integra algunas de las ideas aportadas por Greca y Moreira (2000) sobre diferencias entre modelos mentales y modelos conceptuales. Así mismo incorpora la ya clásica distinción entre conocimiento científico y conocimiento escolar (AAVV, 1994), que en este caso se traduce en la distinción entre modelos científicos (MC) y las representaciones didácticas de esos modelos (RDM) (Galagovsky y Adúriz-Bravo, 2001).

En el recuadro de la izquierda, etiquetado con la palabra alumno, los modelos que aparecen son modelos mentales (MM) (Johnson-Laird, 1983; Holland et al., 1986; Pozo, 1989; Gutiérrez y Ogborn, 1992; Vosniadou, 1994; Greca y Moreira, 1998, 2000). Éstos son idiosincrásicos, personales e internos, y consisten en las representaciones que generamos los individuos durante el funcionamiento cognitivo cuando tratamos de comprender, explicar o predecir el comportamiento de los sistemas con que interaccionamos (Johnson-Laird, 1983; Grosslight, Under, Jay y Smith, 1991; Vosniadou, 1994; Greca y Moreira, 1998; Reiner y Gilbert, 2000; Solsona, Izquierdo y Gutiérrez, 2000).

En el recuadro de la derecha, los modelos que se incluyen son de tipo conceptual, al tratarse de conocimientos de tipo explícito y externo a los individuos, resultado del consenso alcanzado por las comunidades de científicos o de educadores y divulgadores encargados de hacer llegar esa ciencia a los escolares o al público en general. De una parte, los modelos científicos (MC) juegan un papel importante en la estructura y evolución de la ciencia y son parte integral del pensamiento y funcionamiento científico. Se trata de elementos básicos a la hora de fundamentar, adecuar, flexibilizar y evaluar las explicaciones científicas (Halloun, 1996; Gilbert, Boulter y Rutherford, 1998). Harrison y Treagust (2000a) señalan que modelizar es la esencia de pensar y trabajar científicamente, y Gilbert (1993) afirma que la ciencia y sus modelos son inseparables, porque los modelos son los productos de la ciencia y, a la vez, sus métodos y herramientas de trabajo. De otra parte, desde el punto de vista educativo juegan también un papel primordial en la enseñanza de las ciencias, mediatizados por un proceso de transposición didáctica que conduce a las representaciones didácticas de esos modelos (RDM) (Galagovsky y Adúriz-Bravo, 2001). En sus distintas versiones, esas representaciones se utilizan para organizar la comprensión del alumno y como base para delimitar un conocimiento escolar deseable que sirve como punto de referencia externo (Justi y Gilbert, 2000). En ocasiones, esas representaciones se proporcionan directamente en formato declarativo mediante una versión simplificada y adaptada a la edad del modelo científico. En otras, se presentan utilizando recursos e instrumentos retóricos que ayuden a su interiorización, por ejemplo, mediante dibujos, maquetas, modelos mecánicos, metáforas, analogías, simulaciones, paradojas, experimentos mentales, etc.

Como se sugiere en la figura 1, la elaboración y evolución de los modelos mentales en el alumno no es el producto de un proceso de transmisión de significados, sino consecuencia de la evolución cognitiva que resulta de la interacción entre los modelos mentales del alumno y las representaciones didácticas de los modelos científicos (Gutiérrez y Ogborn, 1992; Gutiérrez, 1996; Vosniadou, 1994). En dicha evolución, cada uno de los modelos que se van generando van dejando una huella que no termina por desaparecer a medida que va pasando al estadio siguiente, sino que se mantiene latente dentro del repertorio de modelos que puede utilizar la persona en cada momento. El cambio conceptual, visto de esta forma, se trataría más de la elección de una opción u otra dentro del repertorio de modelos alternativos de que se dispone, que de la sustitución de un modelo por otro (Hewson y Hewson, 1992; Linder, 1993; Maloney y Siegler, 1993; Oliva, 1999; Pozo et al., 1991; Taber, 2001).

Es aquí donde situamos las analogías, como recursos que permiten al alumno construir conocimientos en un dominio dado a partir de su comprensión sobre otro que resulta para él mejor conocido y más familiar.

\section{ENSEÑANZA-APRENDIZAJE POR ANALO- GÍA Y CONSTRUCCIÓN DE MODELOS}

Junto a los modelos mentales y conceptuales, todavía podríamos distinguir, dentro de los primeros, una tercera acepción de la noción de modelo, que resulta básica al hablar de la analogía como recurso didáctico. Se trata de la idea de modelo que introduce Duit (1991) como conjunto de elementos comunes que se aprecian entre los dos sistemas o fenómenos que se comparan durante la construcción de una analogía. A este tercer tipo de modelo lo denominaremos modelo subyacente a la analogía o simplemente modelo de la analogía (MA). En nuestro esquema, el papel de las analogías podría encajar de la forma que se indica en la figura 2.

A la hora de trazar una analogía, un primer paso sería elegir el análogo o situación de anclaje que se utiliza de referencia a la hora de verificar una transferencia de significados hacia la situación o noción objeto que se quiere representar. Se supone que el análogo elegido es bien conocido por el alumno, al menos en aquellas facetas que se pretende utilizar con vistas a ilustrar el objeto. Al evocar la analogía, partimos, pues, de un modelo mental previo que tendrá el alumno sobre la situación objeto $\left(\mathrm{MM}_{\mathrm{i}-1}\right)$, un modelo mental de la situación análoga (MM') y un conjunto de herramientas y representaciones didácticas (RDM) destinadas a estimular el razonamiento analógico en los alumnos. 
Figura 2

El papel del razonamiento analógico en la evolución de los modelos mentales de los alumnos.

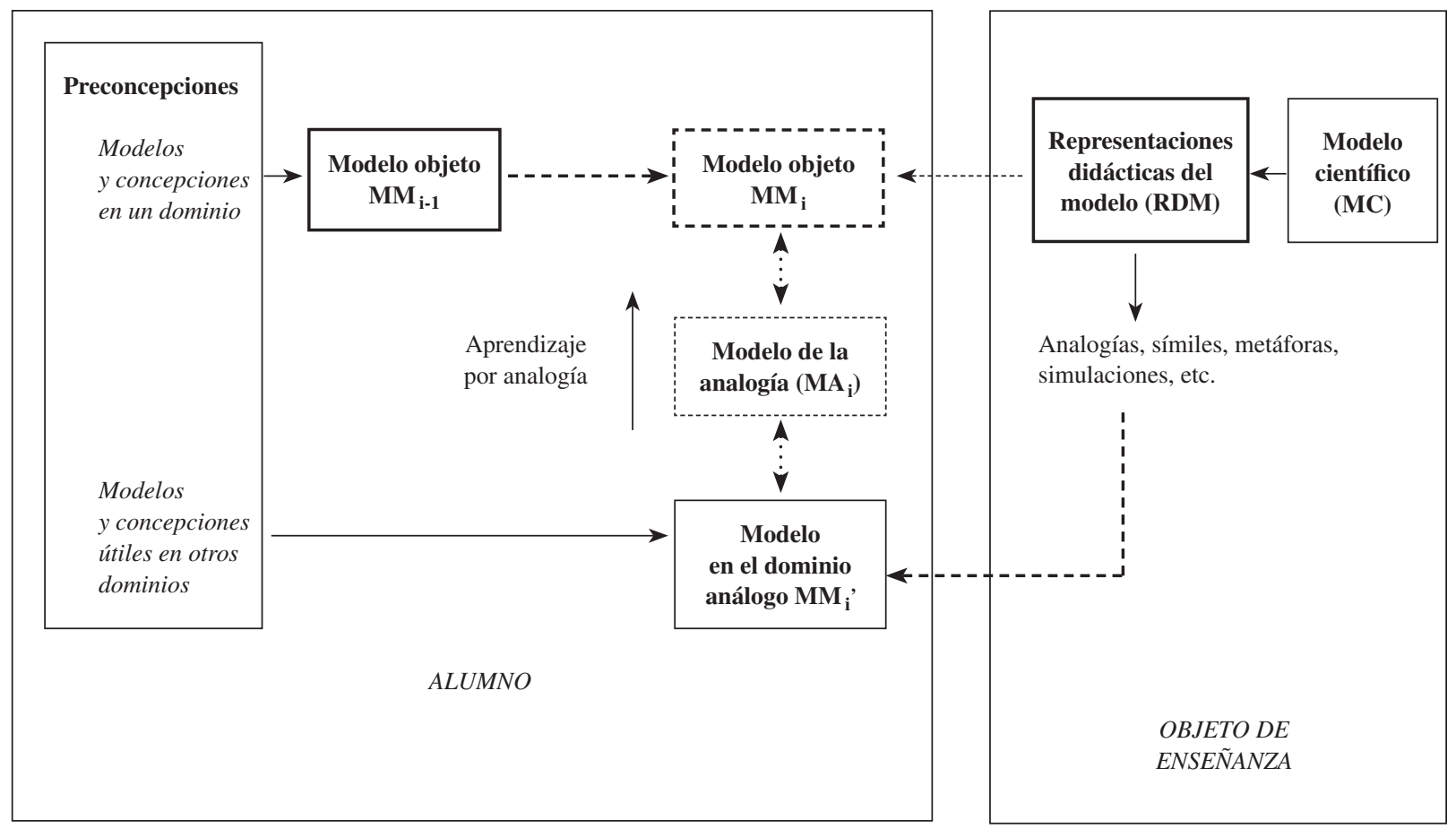

En realidad, todo instrumento dirigido a inducir cualquiera de estos elementos podría ser considerado en sí mismo de naturaleza analógica, por cuanto analógicos son los procesos de transferencia de significados que están en su origen (Van Driel y Verloop, 1999; Gilbert, Boulter y Rutherford, 1998; Harrison y Treagust, 2000a). Así, por ejemplo, cuando utilizamos en las clases de ciencias un modelo molecular de bolas, una maqueta a escala para explicar el sistema solar o globos inflados para ilustrar la forma de distintos orbitales, usamos esos recursos como estímulos para desarrollar el pensamiento analógico de los alumnos.

En este marco, el modelo de la analogía es el fruto de la comparación entre el modelo objeto y el modelo análogo o, lo que es lo mismo, se trata del mensaje que lleva la analogía desde la intencionalidad didáctica con la que se propone. Como señala González-Labra (1997), se trata de un modelo de segundo orden, al tratarse de un proceso que opera ya a partir de otros modelos. La analogía sólo conducirá al conocimiento deseado si el modelo que construye el alumno responde a las expectativas previstas. De ahí que gran parte de las dificultades que surgen en el proceso de aprendizaje mediante analogías -por ejemplo, cuando el alumno aprende el análogo pero no transfiere significados, cuando interpreta la analogía según puntos de vistas inesperados o cuando llevan la analogía demasiado lejos- se podrían interpretar asumiendo que el alumno no ha llegado a construir una representación del modelo que hay de fondo en la analogía o, al menos, que ha construido otro modelo diferente al que el profesor tenía previsto y hubiera deseado.

Veamos a continuación un caso aclaratorio, para lo que recurriremos a una analogía que suele referirse al introducir el modelo atómico de Rutherford en clases de secundaria obligatoria y en mucho libros de texto de ese nivel; se trata de la analogía del átomo como sistema solar $\left(\mathrm{MM}_{\mathrm{i}}{ }^{\prime}\right)$. En este caso, es probable que el alumno disponga ya de una idea de átomo como «esfera sólida», si es que ha estudiado el modelo de Dalton, o como «pudin de pasas» si ha abordado el modelo de Thomson. Cualquiera de estas representaciones mentales podría en principio actuar como modelo de partida $\left(\mathrm{MM}_{\mathrm{i}-1}\right)$, pretendiéndose que el alumno construya un modelo más avanzado de átomo. Éste podría consistir en un núcleo central y electrones que giran alrededor inmersos en un espacio vacío $\left(\mathrm{MM}_{\mathrm{i}}\right)$, para cuyo aprendizaje el profesor podría utilizar una serie de recursos, como presentar la idea de forma esquemática, ofrecer un dibujo aclaratorio y sugerir el símil del átomo como sistema planetario (RDM). Todo ello a pesar de que, como profesores, tenemos en mente un modelo de átomo más complejo como distribución de cargas (MC).

Pues bien, en este contexto, el modelo de la analogía correspondería a aquello que tienen en común los modelos $\mathrm{MM}_{\mathrm{i}}$ y $\mathrm{MM}_{\mathrm{i}}{ }^{\text {'; }}$; es decir, la idea de un cuerpo fijo central, con objetos alrededor dando vueltas atraídos por el cuerpo fijo y, entre ambos, espacio vacío. Éste sería el mensaje que intenta ilustrar la analogía y es lo que Duit (1991) delimita como modelo de la analogía (MA). 
Figura 3

Aprendizaje mediante analogías múltiples consecutivas.

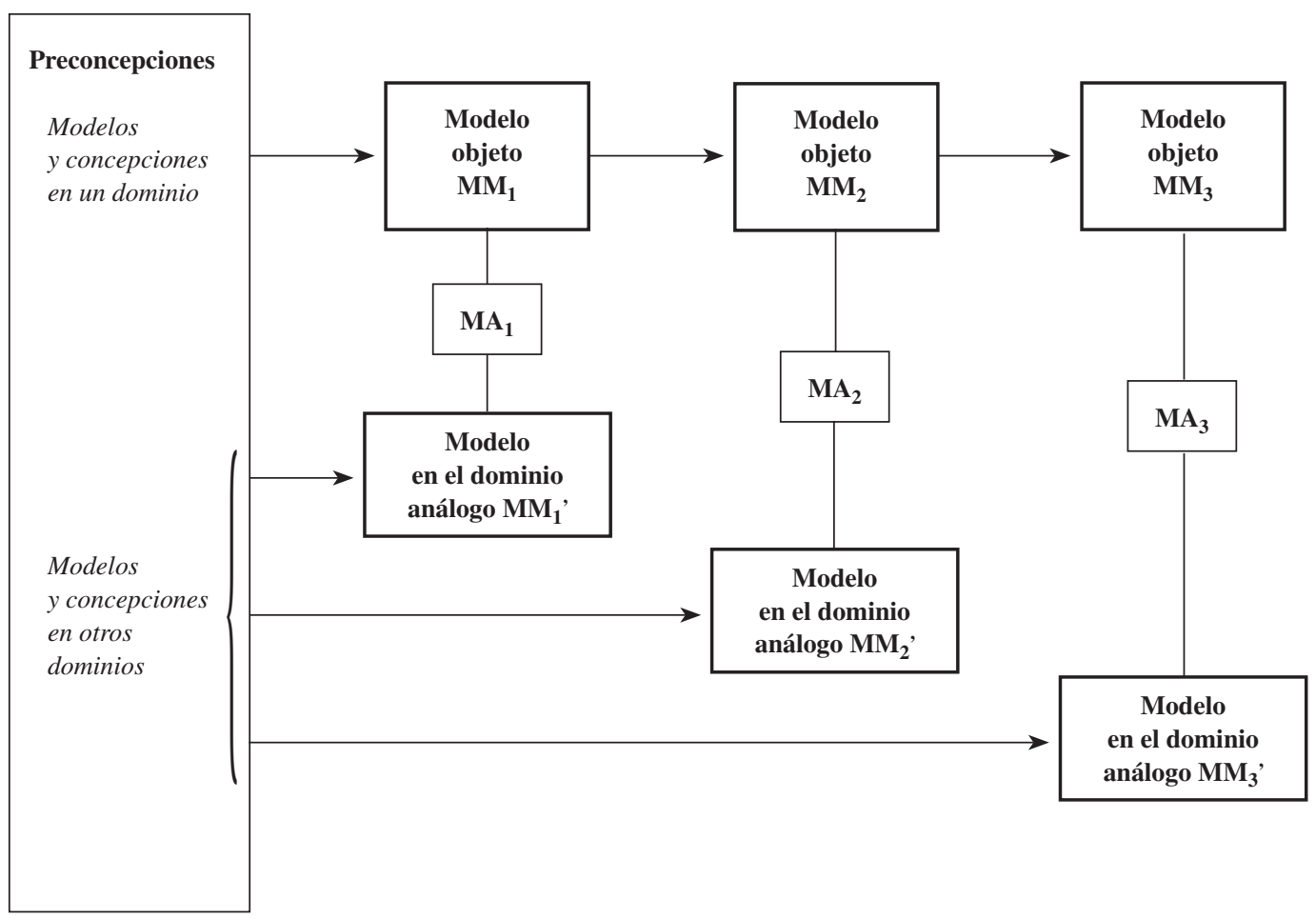

La construcción del modelo de la analogía es una tarea compleja que no se verifica de una forma lineal y unidireccional, sino a través de un proceso interactivo entre el objeto y el análogo en el que tanto el significado que se otorga a la analogía como al modelo que hay detrás se van viendo modificados de una forma paulatina (Oliva et al., 2001). Debido a esta complejidad, surge la conveniencia de recurrir a más de una analogía para ayudar a que el modelo mental que se construya sea próximo al modelo deseable desde el punto de vista de la ciencia escolar (Duit, 1991; Thagard, 1992; Dagher, 1994; Heywood y Parker, 1997; Harrison y Treagust, 2000a, 2000b).

Esta multiplicidad en el uso de analogías debería entenderse en un doble sentido. De un lado, como recurso que permite resaltar y acotar las facetas relevantes del modelo que se pretende ilustrar mediante la comparación entre el objeto y los análogos. De otro, como instrumento que favorece la evolución entre distintos modelos $M_{i}, M_{2} \ldots$ $M_{n}$ si se usa de un modo encadenado a través de diversas analogías sucesivas. Cada una de ellas iría aportando distintos rasgos adicionales al modelo mental del alumno o también modificando aquéllos que resulten inadecuados (Fig. 3). Como ha mostrado Dagher (1994), las analogías pueden tener también una función relevante en el proceso de cambio o evolución conceptual a través de la consecución de pequeños cambios consecutivos que, más tarde, podrían ayudar a precipitar cambios sustanciales en el razonamiento de los alumnos.
Una vez delimitado el modelo cognitivo de alumno por el que nos decantamos a la hora de contemplar el aprendizaje por analogía, queda aún por definir el enfoque que adoptamos en el trabajo a la hora de diseñar y planificar nuestra intervención en el aula. A ello dedicaremos espacio en el siguiente apartado, en el que describiremos un diseño de enseñanza concreto elaborado y aplicado con vistas a favorecer en los alumnos la construcción de un modelo cinético-molecular para la materia a través de analogías.

\section{EL USO DE ANALOGÍAS COMO NÚCLEO VERTEBRADOR DE UNA UNIDAD DIDÁC- TICA SOBRE EL MODELO CINÉTICO-MOLE- CULAR DE LA MATERIA}

La experiencia de enseñanza que desarrollamos estuvo dirigida a estudiantes de $3^{\circ}$ de enseñanza secundaria obligatoria y se prolongó a lo largo de ocho sesiones de clase, de una hora de duración cada una. Los alumnos procedían de dos institutos públicos de ámbito rural. La motivación y el interés de estos alumnos por el aprendizaje eran bastante bajos, y ello se reflejaba en rendimientos académicos bajos por lo general y altos índices de absentismo escolar. Además, el estatus socieconómico de los alumnos implicados era por lo general de nivel mediobajo. Nos encontrábamos, pues, con el reto de enseñar a un alumnado «poco brillante» y escasamente motivado y dispuesto para el estudio. 
Tabla I

Análisis de contenidos para la unidad.

\begin{tabular}{|c|c|c|}
\hline Conceptos & Procedimientos & Actitudes \\
\hline $\begin{array}{l}\text { - Discontinuidad de los sistemas materiales. } \\
\text { - Principios del modelo cinético molecular. } \\
\text { - Cambios de estado. } \\
\text { - Constitución de las mezclas. }\end{array}$ & $\begin{array}{l}\text { - Interpretación de experiencias. } \\
\text { - Elaboración y aplicación de modelos. } \\
\text { - Representación simbólica. } \\
\text { - Desarrollo del razonamiento analógico. }\end{array}$ & $\begin{array}{l}\text { - Valoración de la utilidad de los modelos } \\
\text { científicos en la interpretación y predicción } \\
\text { de hechos. } \\
\text { - Reconocimiento de sus limitaciones. }\end{array}$ \\
\hline
\end{tabular}

El diseño y desarrollo de la unidad didáctica se efectuó de acuerdo con la propuesta realizada por Sánchez-Blanco y Valcárcel (1993). Por problemas de espacio, no podemos ofrecer un análisis completo de todo el proceso seguido. No obstante, sí nos parece conveniente aportar algunas pistas para que el lector adquiera una primera idea de la unidad didáctica elaborada y aplicada, y le sirva también para contextualizar los datos que más tarde se ofrecen. Por ejemplo, como parte del proceso de análisis científico realizado, nos parece interesante aportar el resultado del análisis de contenidos, el cual se recoge en la tabla I.

Igualmente, como parte del análisis didáctico, aportamos algunos comentarios acerca de los conocimientos de partida de los alumnos que intervinieron. Estos, en sus cursos anteriores, no habían abordado nociones sobre estructura y propiedades de la materia ni sobre química. Sólo habían estudiado contenidos correspondientes a las ciencias naturales y algunas nociones de física. Por tanto, se trataba de la primera vez que estudiaban nociones relativas al modelo cinético-molecular de la materia, al menos dentro del currículo académico.

Si bien, por razones que se señalan más adelante, no se utilizó ninguna prueba previa propiamente dicha para analizar el nivel de partida, los prolegómenos de la unidad nos dieron la oportunidad de comprobar in situ cuál era el modelo de materia que mantenía el conjunto de participantes. En efecto, a través de algunas preguntas preliminares planteadas en clase, pudimos comprobar cómo globalmente todos ellos mantenían una visión macroscópica de la materia, basada en un nivel descriptivo de los fenómenos que se les planteaba, sin alusiones significativas a un modelo microscópico basado en moléculas y huecos o espacio vacío. Este dato resulta bastante lógico si se tiene en cuenta que la idea de discontinuidad de la materia, en términos atomistas, no es una noción que se adquiera fruto del desarrollo espontáneo de las personas sin una intervención didáctica intencionada (Benarroch, 2000). Por otra parte, concuerda bastante bien con el dato que obtuvimos en un trabajo anterior con alumnos algo mayores, concretamente procedentes del extinto curso de $2^{\circ}$ de BUP (Oliva e Iglesias, 1989). En dicho estudio pudimos apreciar cómo los alumnos suelen hacer muy escaso uso de un modelo de partículas a la hora de interpretar o predecir fenómenos relativos al comportamiento físico de la materia.

Consecuencia de una reflexión conjunta del análisis científico y del análisis didáctico efectuado, surgieron los objetivos perseguidos en la unidad. Éstos fueron los siguientes:

a) Contemplar una visión explicativa de la materia basada en un nivel microscópico o molecular.

b) Conocer y comprender los postulados básicos del modelo cinético molecular de la materia.

c) Interpretar, según la teoría cinético molecular, las propiedades de las sustancias en diferentes estados de agregación y fenómenos como las mezclas, separaciones y cambios de estado.

d) Predecir fenómenos y propiedades empleando el modelo cinético molecular.

e) Valorar el sentido, la utilidad y las limitaciones de los modelos en la ciencia.

El referente externo del modelo a construir consistía en una versión cualitativa del modelo cinético-molecular. En dicha versión, las moléculas eran representadas unas veces mediante círculos o esferas, y otras mediante análogos extraídos de la vida diaria: lentejas, palomitas, pelotas de tenis, etc. La tabla II recoge de forma escueta el nivel de conceptualización alcanzado en los contenidos tratados.

Durante el diseño y la aplicación de la unidad didáctica que elaboramos, el uso de analogías se convirtió en un recurso central dentro del repertorio empleado. Por ejemplo, actuó como núcleo vertebrador de la estructura de la unidad y, también, como instrumento dirigido a «enganchar» a los alumnos motivándoles y ayudándoles a construir modelos abstractos a partir de nociones próximas a la vida diaria.

El enfoque didáctico seguido al trabajar con analogías podría encuadrase dentro de un marco más amplio fundamentado y descrito en otro lugar (Oliva et al., 2001) basado, a su vez, en trabajos de autores como Dagher (1995), Duit (1991), Glyn (1991), Harrison y Treagust (2000a), etc. En realidad, dicho marco sólo fue aplicado parcialmente en el desarrollo de la unidad didáctica, ya que, de hecho, fue gestándose durante el diseño de la misma, en paralelo con el desarrollo de la investigación. Consecuencia del proceso de evaluación realizado, que más adelante detallaremos, ha sido el diseño de una segunda generación de materiales en los que las bases 
de dicho marco de trabajo se han visto notablemente reforzadas.

El enfoque adoptado se podría concretar a través de los siguientes puntos:

- La presentación de la situación objeto de estudio, es decir, de los fenómenos que se pretendían estudiar y de la versión didáctica del modelo científico que los explicaba. Pensábamos que, en esta fase, el alumno necesitaría disponer de una referencia que diera sentido al contenido objeto de estudio, así como también explicitar las ideas previas que se mantenían al respecto.

- La introducción de una analogía, o de varias, que presentasen un modelo común con el objeto.

- El estudio de las semejanzas existente entre el objeto y el análogo, a través del trazado de relaciones entre los elementos de uno y otro.

- El establecimiento de diferencias que pusiesen de manifiesto las limitaciones de las analogías. Además de aclarar cuál es la validez de la analogía, creíamos que la búsqueda de caracteres diferenciadores nos ayudaría a esclarecer las características del modelo.

- La aplicación de la analogía en actividades de interpretación y predicción de fenómenos, con la pretensión de afianzar el modelo y los conceptos asociados. Se esperaba con ello favorecer la transferencia de conocimientos entre campos distintos, así como evaluar la utilidad de la analogía y validar el modelo construido.

- La profundización en el modelo mediante actividades de síntesis de conclusiones y el planteamiento de actividades destinadas a introducir cambios sobre aquellas concepciones alternativas que se habían detectado al principio. La superación de estas ideas, pensábamos que resultaría ahora más fácil una vez que el alumno dispone ya, en su estructura de conocimientos, de un modelo coherente en el que enmarcar las nuevas ideas.

Para más detalles acerca de la secuencia seguida y de los tipos de actividades con las que trabajamos, puede consultarse la tabla III así como los dos ejemplos de actividades que se muestran en el anexo. La tabla III se ha elaborado aprovechando el formato utilizado por Sánchez-Blanco, Pro y Valcárcel (1997) a la hora de sintetizar la estructura y secuencia de una unidad didáctica.

Desde el punto de vista de la metodología empleada en la intervención, adoptamos una metodología didáctica que propiciaba un entorno activo y participativo para los alumnos, partiendo de sus concepciones iniciales y tomando como modelo un aprendizaje de tipo significativo. Las actividades se realizaron normalmente con los alumnos distribuidos en pequeños grupos dejando un espacio de tiempo al final para una puesta en común.

En cuanto al proceso de evaluación seguido se analizaron, entre otras cosas, aspectos como el nivel final de utilización del modelo cinético-molecular en tareas de interpretación y predicción de fenómenos, el grado de comprensión de algunas de las analogías más representativas incluidas en la secuencia y las actitudes y opiniones de los alumnos acerca de las actividades que habían desarrollado en clase, con especial atención a aquéllas que hacían referencia al trabajo con analogías.

Tabla II

Nivel de conceptualización adoptado como referente en las representaciones didácticas del modelo cinético-molecular.

\begin{tabular}{|l|l|}
\hline \multicolumn{1}{|c|}{ Sistema } & \multicolumn{1}{c|}{ Constitución } \\
\hline $\begin{array}{l}\text { La materia } \\
\text { en general }\end{array}$ & $\begin{array}{l}\text { La materia está formada por moléculas y espacio vacío. Entre las moléculas existen fuerzas atractivas y repulsivas. Si las fuerzas } \\
\text { atractivas son pequeñas, éstas se alejarán debido a su movimiento. Si dos moléculas se acercan mucho, debido a las fuerzas } \\
\text { repulsivas se repelerán. }\end{array}$ \\
\hline Gaseoso & $\begin{array}{l}\text { Las moléculas están muy separadas, unas diez veces más que en el estado líquido, y se mueven desplazándose aleatoriamente } \\
\text { de un lugar a otro pudiéndose separar indefinidamente. }\end{array}$ \\
\hline Líquido & $\begin{array}{l}\text { Las moléculas están desordenadas y las distancias son aproximadamente iguales que en estado sólido. Pueden desplazarse de } \\
\text { un lugar a otro, pero no pueden separarse indefinidamente. }\end{array}$ \\
\hline Sólido & $\begin{array}{l}\text { Las moléculas tienen movimiento vibratorio y las distancias entre moléculas son del orden del diámetro molecular. En los lla- } \\
\text { mados sólidos cristalinos, las moléculas están dispuestas de forma ordenada. }\end{array}$ \\
\hline Mezcla & $\begin{array}{l}\text { Sustancias diferentes tienen moléculas distintas. En una mezcla existen dos tipos de sustancias o, lo que es lo mismo, dos tipos } \\
\text { de moléculas. En las llamadas disoluciones, las moléculas están mezcladas uniformemente; en las heterogéneas, no. }\end{array}$ \\
\hline
\end{tabular}




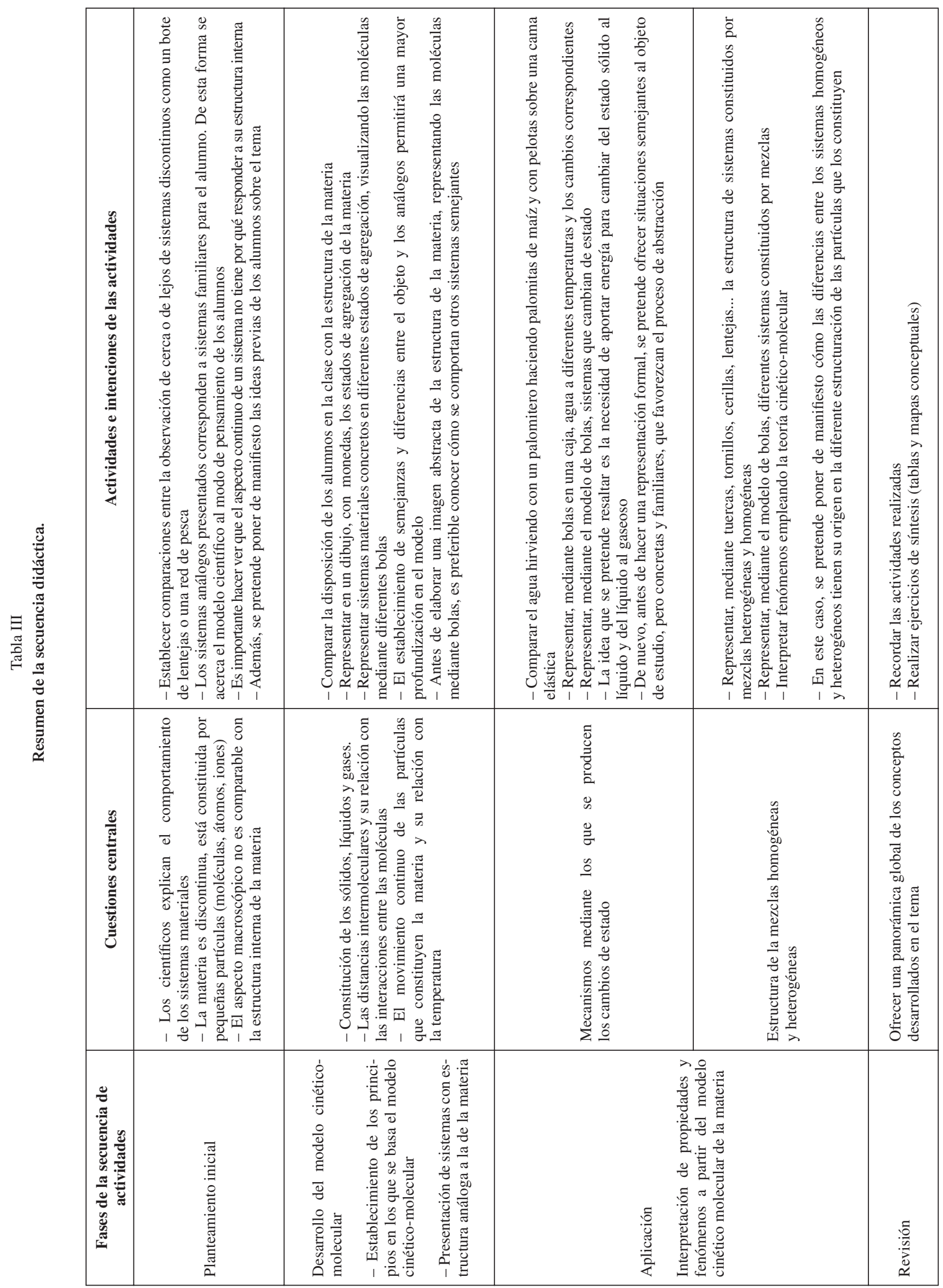




\section{PROPÓSITOS Y DISEÑO DE LA INVESTIGA- CIÓN}

Aunque, como ya hemos indicado, el ámbito de evaluación contemplado era más general, en este estudio nos limitamos a contrastar el grado de madurez alcanzado por los modelos mentales de los alumnos que fueron enseñados utilizando analogías como hilo conductor. Dejamos, para futuros trabajos, la evaluación de los otros temas señalados.

Con objeto de establecer una referencia externa que facilitase el balance final que se pretendía realizar, recurrimos a un grupo de control integrado por alumnos que habían sido enseñados en el tema mediante una secuencia de contenidos y una dinámica de aula muy similares pero sin incluir analogías en su desarrollo. La modificación se efectuó con extremo cuidado para no introducir diferencias sustanciales en los contenidos tratados con respecto al otro grupo. En ambos casos, el número de sesiones dedicadas al desarrollo de estos contenidos fue el mismo, concretamente ocho como ya hemos dicho.

Como instrumento de evaluación utilizamos un cuestionario destinado a investigar los modelos activados por los alumnos durante la interpretación y predicción de fenómenos físicos (estados de la materia, cambios de estado, difusión, etc.). El cuestionario estaba formado por seis tareas cuya estructura y contenido se sintetizan en la tabla IV. Un ejemplo de tarea se recoge en el anexo II.

Este cuestionario se administró algunas semanas después de completar el tema objeto de análisis y tenía por finalidad evaluar: $a$ ) en qué medida los alumnos identificarían diferencias sustanciales entre el espacio intermolecular existente en un líquido y en un gas; y $b$ ) si los alumnos otorgarían o no movimiento intrínseco a las moléculas, concretamente de un gas, logrando así explicar fenómenos como la difusión.

Tabla IV

Esquema del contenido de la prueba final de interpretación de fenómenos mediante la TCM.

\begin{tabular}{|c|c|c|c|c|c|}
\hline Tarea & Contenido tratado & Contexto de la tarea & $\begin{array}{c}\text { Formato } \\
\text { de la pregunta }\end{array}$ & Pregunta formulada & $\begin{array}{l}\text { Respuesta esperada desde } \\
\text { el punto de vista científico }\end{array}$ \\
\hline 1 & $\begin{array}{l}\text { Diferencias en el es- } \\
\text { pacio intermolecular } \\
\text { de un líquido y de } \\
\text { un gas. }\end{array}$ & $\begin{array}{l}\text { Ebullición de agua en } \\
\text { una olla a presión total- } \\
\text { mente repleta. }\end{array}$ & $\begin{array}{l}\text { Predicción del fenóme- } \\
\text { no: opción múltiple. } \\
\text { Justificación de la res- } \\
\text { puesta: abierta. }\end{array}$ & $\begin{array}{l}\text { ¿Es posible, en esas } \\
\text { condiciones, el paso de } \\
\text { todo el líquido al estado } \\
\text { de vapor? }\end{array}$ & $\begin{array}{l}\text { Los gases ocupan más volu- } \\
\text { men que los líquidos, ya que } \\
\text { sus moléculas se encuentran } \\
\text { mucho más distantes entre sí. }\end{array}$ \\
\hline 2 & $\begin{array}{l}\text { Movilidad de las } \\
\text { moléculas, concreta- } \\
\text { mente en un gas. }\end{array}$ & $\begin{array}{l}\text { El aroma que perci- } \\
\text { bimos de un tarro de } \\
\text { colonia. }\end{array}$ & $\begin{array}{l}\text { Predicción del fenóme- } \\
\text { no: opción múltiple. } \\
\text { Justificación de la res- } \\
\text { puesta: abierta. }\end{array}$ & $\begin{array}{l}\text { ¿Cuándo te llega antes } \\
\text { el olor?: ¿en verano o } \\
\text { en invierno? }\end{array}$ & $\begin{array}{l}\text { La mayor temperatura del } \\
\text { verano favorece el proceso } \\
\text { de difusión del gas al moverse } \\
\text { sus moléculas más rápida- } \\
\text { mente. }\end{array}$ \\
\hline 3 & $\begin{array}{l}\text { Diferencias en el es- } \\
\text { pacio intermolecular } \\
\text { de un líquido y de } \\
\text { un gas. }\end{array}$ & $\begin{array}{l}\text { Dos recipientes igua- } \\
\text { les: uno lleno de agua } \\
\text { líquida y otro de vapor } \\
\text { de agua. }\end{array}$ & $\begin{array}{l}\text { Explicación del fenóme- } \\
\text { no presentado: respuesta } \\
\text { abierta. }\end{array}$ & $\begin{array}{l}\text { ¿Por qué el que está lle- } \\
\text { no de agua pesa más? }\end{array}$ & $\begin{array}{l}\text { El recipiente con agua líquida } \\
\text { contiene mayor número de } \\
\text { moléculas, ya que en ella el } \\
\text { espacio intermolecular es } \\
\text { mucho menor. }\end{array}$ \\
\hline 4 & $\begin{array}{l}\text { Movilidad de las mo- } \\
\text { léculas, concretamente } \\
\text { en un gas. }\end{array}$ & Expansión de un gas. & $\begin{array}{l}\text { Explicación del fenóme- } \\
\text { no presentado: respuesta } \\
\text { abierta. }\end{array}$ & $\begin{array}{l}\text { ¿Por qué un gas tiende } \\
\text { siempre a ocupar todo el } \\
\text { volumen del recipiente } \\
\text { que lo contiene? }\end{array}$ & $\begin{array}{l}\text { La movilidad intrínseca } \\
\text { y la libertad de las moléculas } \\
\text { posibilita su difusión a través } \\
\text { del recipiente. }\end{array}$ \\
\hline 5 & $\begin{array}{l}\text { Diferencias en el es- } \\
\text { pacio intermolecular } \\
\text { de un líquido y de } \\
\text { un gas. }\end{array}$ & $\begin{array}{l}\text { Se intenta comprimir un } \\
\text { poco de agua mediante } \\
\text { una jeringa con la salida } \\
\text { obstruida. }\end{array}$ & $\begin{array}{l}\text { Explicación del fenóme- } \\
\text { no presentado: respuesta } \\
\text { abierta pero advirtiendo } \\
\text { que tenían que usar el } \\
\text { MCM. }\end{array}$ & $\begin{array}{l}\text { ¿Por qué no es posible } \\
\text { comprimir apenas el } \\
\text { agua y sí un poco de } \\
\text { aire? }\end{array}$ & $\begin{array}{l}\text { En el agua líquida, el espacio } \\
\text { intermolecular es pequeño, } \\
\text { mientras en el aire es mucho } \\
\text { mayor. }\end{array}$ \\
\hline 6 & $\begin{array}{l}\text { Movilidad de las mo- } \\
\text { léculas, concretamente } \\
\text { en un gas. }\end{array}$ & $\begin{array}{l}\text { Difusión de un gas des- } \\
\text { de un recipiente a otro } \\
\text { vacío conectados ambos } \\
\text { mediante un tubo. }\end{array}$ & $\begin{array}{l}\text { Explicación del fenóme- } \\
\text { no presentado: respuesta } \\
\text { abierta pero advirtiendo } \\
\text { que tenían que usar el } \\
\text { MCM. }\end{array}$ & $\begin{array}{l}\text { ¿Por qué el gas se } \\
\text { reparte entre ambos } \\
\text { recipientes? }\end{array}$ & $\begin{array}{l}\text { La movilidad intrínseca } \\
\text { y la libertad de las moléculas } \\
\text { posibilita su difusión de un } \\
\text { recipiente a otro. }\end{array}$ \\
\hline
\end{tabular}


La evaluación se llevó a cabo a partir de una muestra de 128 estudiantes de $3^{\circ}$ de ESO provenientes de seis grupos de clase, cuatro de ellos de un instituto de secundaria, al que llamaremos $A$, y otros dos de un instituto distinto, también de secundaria, al que llamaremos $B$. Todos los grupos participantes del centro $A$ estaban impartidos por la misma profesora, y todos los del centro $B$ lo estaban también por el mismo profesor.

El diseño utilizado fue de tipo casi experimental. La distribución de grupos clase a los respectivos grupos, experimental y de control, se realizó según un diseño pareado que tenía en cuenta las calificaciones escolares en el curso anterior, de manera que finalmente hubiera un cierto equilibrio en esa variable. De esta forma, el grupo experimental quedó constituido por dos grupos de clase del centro $A$ y uno del centro $B$, con un total de 65 estudiantes. Mientras tanto, el grupo de control quedó formado también por dos grupos de clase del centro $A$ y uno del centro $B$, esta vez contabilizando un total de 63 estudiantes. Aunque el nivel académico de partida, medido a través de las mencionadas calificaciones, resultó algo superior en el grupo de control que en el experimental, las diferencias no llegaron a ser estadísticamente significativas $\mathrm{y}$, en todo caso, operaban en contra de la hipótesis a contrastar, que era la de una supuesta superioridad de los modelos mentales finales de los alumnos del grupo experimental con respecto a los de sus homólogos del grupo de control.

Para el control de la variable alumno, pensamos en principio tomar también como referencia el dato de los modelos mentales disponibles inicialmente -antes de la experiencia- sobre el comportamiento de la materia. Sin embargo, la homogeneidad observada en esos modelos de partida a través del sondeo previo realizado, cuyas conclusiones encajaban bien con los precedentes de que disponíamos (Oliva e Iglesias, 1989), nos hizo desistir de tal opción. En efecto, dicho sondeo nos sirvió, como ya hemos comentado en otro lugar, para comprobar que mayoritariamente el alumnado partía de un modelo macroscópico de materia, mostrándose, por tanto, este factor como un instrumento de escaso valor para detectar posibles diferencias en la variable alumno.

Por todo ello, y basándonos en las consideraciones expuestas, parece lógico presuponer que, inicialmente, los alumnos experimentales y de control formasen parte de la misma población estudiantil y que, por tanto, no deberían existir diferencias sustantivas de partida entre ambos. De ahí que cobrase también sentido esperar que cualquier eventual diferencia que operase al final entre los modelos mentales de ambos grupos respondiese, al efecto del tratamiento didáctico empleado, esto es, al uso de las analogías como recurso didáctico.

\section{RESULTADOS}

\section{Análisis cualitativo de las respuestas}

Con objeto de revisar las respuestas de los alumnos establecimos sistemas de categorías para las distintas tareas, los cuales fueron cambiando y siendo objeto de diversas modificaciones a medida que el estado de la investigación fue avanzando. Desde el principio combinaba criterios cualitativos y cuantitativos de proximidad al punto de vista científico, pero inicialmente el número de categorías consideradas era demasiado amplio, variaba de unas tareas a otras y no mostraban un claro orden jerárquico entre ellas. Todo ello no hacía sino dificultar el proceso de clasificación de respuestas y la comparación de resultados entre distintas tareas.

Con posterioridad, algunas de las categorías fueron agrupadas según la similitud observada entre ellas, hasta que finalmente logramos definir una escala ordinal de cinco niveles que nos pareció bastante operativa. Dicha escala tenía la ventaja de reflejar muchas de las características del itinerario evolutivo que algunas autoras han perfilado para la variedad de interpretaciones de los alumnos en este campo (Benarroch, 2000). De hecho, nuestro sistema de categorización fue semejante al de la citada autora aunque aparecían algunas diferencias importantes como veremos después.

A continuación mostramos los criterios utilizados en la clasificación de respuestas según los distintos niveles, ilustrándolos con ejemplos concretos extraídos de respuestas aportadas a las tareas 3 y 6 . La tarea número 3 trataba de evaluar la visión de los alumnos acerca de las diferencias en el espacio intermolecular entre un líquido y un gas, cuestionándoles sobre la razón de que un recipiente lleno de agua líquida pese más que otro que contiene vapor de agua (Anexo II). Mientras tanto, la número 6 analizaba la idea de movimiento molecular preguntando las razones de los fenómenos de difusión de los gases. Los criterios de clasificación fueron los siguientes:

Nivel 1. Contestaciones en blanco o, también, respuestas que presentaban una imagen continua de la materia con explicaciones claramente desviadas de las que utiliza la ciencia para interpretar el fenómeno. En otras ocasiones, las explicaciones aportadas eran poco elaboradas o incluso triviales. Ejemplos:

- El líquido pesa más porque ambos tienen el mismo volumen pero no el mismo peso. Los gases no pesan. (Tarea 3)

- Porque uno está lleno y el otro vacío, así que, cuando se abra la llave, ambos [recipientes] se ponen al mismo nivel. (Tarea 6)

Nivel 2. Respuestas con un cierto grado de elaboración, que podrían considerarse parcialmente correctas desde un punto de vista macroscópico, pero que omitían referencias a una interpretación discontinua de la materia. Ejemplos:

- Porque, en los líquidos, la materia está más comprimida que en los gases. Los líquidos siempre tienen mayor densidad. (Tarea 3)

- El gas está comprimido en uno de los recipientes. Por tanto, cuando abramos la llave, se repartirá entre ambos porque tenderá a ocupar todo el espacio que le dejemos. (Tarea 6) 
Nivel 3. Respuestas que, haciendo uso de una visión discontinua de la materia, incurrían en concepciones alejadas del punto de vista de la ciencia escolar. En ocasiones, estas desviaciones se basaban en proyecciones de las propiedades del mundo macroscópico sobre el microscópico. Ejemplos:

- Porque las moléculas de agua líquida pesan más que las de vapor. (Tarea 3)

- Porque el vacío que existe en el otro recipiente «tira» de las moléculas del recipiente lleno. (Tarea 6).

Nivel 4. Interpretación discontinua de la materia bastante próxima al modelo objeto, con algunas imprecisiones en el lenguaje o que omitían aspectos relevantes como el movimiento de las partículas. En ocasiones la idea de movimiento se introducía sólo como un fenómeno transitorio y ordenado. Ejemplos:

- Las moléculas están más próximas entre sí en A [recipiente con agua] que en B [recipiente con vapor]. (Tarea 3)

- Porque las moléculas del gas se empiezan a mover separándose hasta ocupar los dos recipientes. (Tarea 6)

Nivel 5. Respuestas que mostraban un alto grado de aproximación a la ciencia escolar en el marco expuesto para la teórica cinético-molecular en la tabla II.

\section{Ejemplos:}

- En el recipiente A [agua líquida], las moléculas se mueven poco y están todas muy juntas. En el recipiente B [vapor de agua], las moléculas se mueven mucho y por ello están más separadas. (Tarea 3)

- Ya que las moléculas de un gas están siempre en movimiento de un lado para otro, tendiendo por ello a ocupar todo el espacio posible. (Tarea 6)

En ocasiones, nos encontramos con serias dudas a la hora de categorizar o no ciertas respuestas de los alumnos dentro de este nivel 5, que era el máximo posible de la escala. Así, en el caso que acabamos de exponer de la tarea 3, algunos alumnos aludían a una supuesta menor movilidad de las moléculas en el estado líquido que en el gaseoso, lo cual resulta incierto si el término movilidad se interpreta como velocidad o energía cinética y la temperatura es la misma en ambos casos. No obstante, de un lado, la igualdad de la temperaturas no era algo que se advirtiese expresamente en la tarea planteada. De otro, las expresiones empleadas por los alumnos cuando hablaban de «moverse mucho» o «moverse poco», también podían interpretarse en términos de capacidad o limitación de desplazamiento de las moléculas y no tanto de velocidad. En estos casos, y ante la duda, preferimos evaluar las respuestas de forma «generosa» situándolas en el nivel 5 o máximo posible. La condición que pedimos para ello es que, cuanto menos, el modelo representado cumpliese con los dos criterios básicos que caracterizaban la RDM, esto es, la existencia de diferencias sustanciales en el espacio intermolecular de un líquido y de un gas, y la agitación o movimiento molecular.
La diferencia esencial entre la clasificación empleada por Benarroch y la aquí utilizada estriba en la naturaleza del nivel 2. En aquel caso, se contemplaba como nivel 2 un tipo de explicación en la que, según dicha autora, aparecían «modelos de materia que siguen siendo continuos, pero que se ven enriquecidos con elementos percibidos (burbujas, huecos, pompitas, etc.)» (Benarroch, 2000, p. 238). Sin embargo, este tipo de explicación fue prácticamente inexistente en nuestro trabajo y, por ello, no fue tenida en cuenta. En su lugar, en cambio, encontramos un grupo de explicaciones macroscópicas que presentaban un grado de elaboración superior a las del nivel 1, probablemente influidas por el aprendizaje anterior y el lenguaje adquirido en otros temas del currículo que recurren a explicaciones científicas basadas en una perspectiva macroscópica para explicar determinados fenómenos. En efecto, en algunos temas de la ciencia (presión en líquidos, calor y temperatura, etc.) se estudian propiedades de la materia desde un punto de vista macroscópico, por constituir formas de saber epistemológicamente ligadas a etapas históricas anteriores a la generalización de la formulación y aceptación del modelo cinético-molecular. Es posible, por tanto, que algunos alumnos hayan logrado desarrollar argumentos más sofisticados que el resto al interpretar determinados fenómenos físicos, aun cuando sus explicaciones se sigan moviendo en un terreno alejado de una ciencia atomista o molecular.

Las tareas fueron revisadas por tres jueces con objeto de triangular opiniones. Se intentó, en primera instancia, que la asignación de niveles a las distintas respuestas fuera el producto de la unanimidad entre los tres jueces. Ello fue posible en la mayoría de casos. En los restantes, se recurrió, en su defecto, a un criterio de consenso entre al menos dos jueces.

\section{Estudio comparativo entre grupos}

Durante el desarrollo de investigación surgieron dos dilemas a la hora de decidir la forma de analizar los datos: el estatus métrico asignado a los mismos (ordinal o de intervalo) y la forma de tratar éstos a lo largo de las distintas tareas (análisis separado o análisis conjunto). En principio nos pareció conveniente asignar un estatus ordinal a las medidas -estableciendo un sistema de puntuaciones entre 1 y 5 - y recurrir a un tratamiento separado de las distintas tareas. Siguiendo estos criterios, la tabla $\mathrm{V}$ presenta las medianas que arrojaban cada una de las tareas, así como los promedios de rangos alcanzados en cada grupo, una vez que los datos de ambos grupos fueron combinados y ordenados en una misma serie, de menor a mayor.

Antes de entrar de lleno en el análisis de los datos conviene matizar que las dos primeras tareas demandaban la predicción e interpretación de un fenómeno, las dos siguientes sólo interpretación, y las dos últimas también sólo interpretación pero advirtiendo además la necesidad de utilizar el modelo cinético molecular en sus explicaciones. Por ello, era de esperar que en ese orden disminuyese el grado de dificultad de las tareas planteadas y mejorase el estatus de los modelos activados a través de ellas. 
Entrando ya en el análisis, hemos de señalar, en primer lugar, que los promedios obtenidos variaron en ambos grupos de unas tareas a otras, siendo bajos en las dos primeras tareas, y más altos normalmente en las restantes, de acuerdo más o menos con los resultados que se preveían. La excepción a esta regla la encontramos en la tarea 6 , cuyos resultados mostraron un nivel de dificultad mayor del esperado.

En segundo lugar, hemos de indicar que los resultados finales alcanzados, tanto en el grupo experimental como en el de control, pueden considerarse globalmente satisfactorios, con promedios de utilización de modelos mentales próximos al modelo objeto de la enseñanza en dos de las tareas ( 4 y 5 ) y modelos con estatus intermedio en otras dos (tareas 3 y 6). Todo ello a pesar de que el nivel académico de los alumnos implicados era por lo general bastante bajo, como ya hemos indicado. Ello indica un cierto grado de éxito en el diseño ensayado de cara a hacer evolucionar los modelos mentales de los alumnos, desde aquéllos macroscópicos de sentido común a otros más próximos al modelo cinético-molecular. Por tanto, los resultados obtenidos pueden considerarse ciertamente alentadores.

En tercer lugar, y entrando en las diferencias, es preciso observar que los valores de las medianas se mostraron algo superiores en el grupo experimental que en el de control, a lo largo de las tres primeras tareas. Así, pues, aunque el alumnado del grupo experimental partiese de un nivel académico globalmente algo inferior al del grupo control, finalmente manifestó un mayor nivel en sus explicaciones basadas en el modelo cinético-molecular, al menos en algunas tareas. No obstante, en ningún caso, las diferencias detectadas fueron significativas estadís- ticamente. Por tanto, aunque los resultados obtenidos parecen apuntar hacia una cierta superioridad en las ideas de los alumnos que fueron enseñados a través de analogías con respecto a los que no lo fueron, los datos aportados no pueden considerarse concluyentes.

El hecho, sin embargo, de que las diferencias obtenidas fueran pequeñas, pero siempre a favor del grupo experimental, nos hizo pensar que, tal vez, la acumulación de estas pequeñas diferencias pudiera convertirse en significativas si se combinasen en una prueba única. Pero la acumulación de evidencias a través de diversas pruebas estadísticas no puede realizarse directamente a través de los valores de $\alpha$, ya que éstos no son directamente acumulables. Sí lo son, en cambio, los valores del estadístico $\chi^{2}$, en los que siempre es posible transformar aquéllos mediante una ecuación logarítmica de $\alpha^{2}$, con $2 k$ grados de libertad, siendo $k$ el número de valores $\alpha$ que se acumulan.

Cuando se combina el efecto estadístico de las tres primeras tareas resulta un $\chi^{2}=13,045(\mathrm{~g} .1 .=2 \times 3=6)$, que es estadísticamente significativo al nivel de $\alpha<0,05$. Así, pues, los datos obtenidos permiten rechazar la hipótesis nula y aceptar la hipótesis alternativa, si bien las diferencias detectadas eran pequeñas.

Un aspecto importante a comentar en este sentido es que las diferencias observadas se manifestaran solamente en tareas con mayor grado de dificultad. De hecho, se detectaron en las dos tareas más complejas (1 y 2) y en una de las dos siguientes (3). Este hecho sugiere que sea ante situaciones y tareas de mayor complejidad, cuando las eventuales diferencias que se pretendían obtener apareciesen entre alumnos enseñados o no mediante analogías.

Tabla V

Comparación de resultados para las distintas tareas en el grupo experimental y de control.

\begin{tabular}{|c|c|c|c|c|c|}
\hline \multirow{2}{*}{ Tarea } & \multicolumn{2}{|c|}{ Mediana (1-5) } & \multicolumn{2}{c|}{$\begin{array}{c}\text { Rango medio de menor } \\
\text { a mayor (1-128) }\end{array}$} & $\begin{array}{c}\alpha \\
\text { Prueba U de } \\
\text { Mann-Whitney }\end{array}$ \\
\cline { 2 - 5 } & $\begin{array}{c}\text { Grupo } \\
\text { de control }\end{array}$ & $\begin{array}{c}\text { Grupo } \\
\text { experimental }\end{array}$ & $\begin{array}{c}\text { Grupo } \\
\text { de control }\end{array}$ & Grupo experimental & \\
\hline 1 & 1 & 2 & 59,4 & 69,5 & 0,11 \\
\hline 2 & 2 & 3 & 59,4 & 69,4 & 0,10 \\
\hline 3 & 2 & 4 & 60,0 & 68,8 & 0,15 \\
\hline 4 & 4 & 4 & 64,4 & 64,6 & 0,97 \\
\hline 5 & 4 & 4 & 63,4 & 65,6 & 0,90 \\
\hline 6 & 3 & 3 & 62,3 & 66,6 & 0,49 \\
\hline
\end{tabular}




\section{DISCUSIÓN Y CONCLUSIONES}

Antes de entrar en valoraciones del diseño didáctico concreto utilizado en este trabajo, querríamos comentar, a modo de resumen, algunos aspectos referentes al marco teórico adoptado a la hora de contemplar el aprendizaje de modelos a través de analogías.

En primer lugar, queremos comentar que en este trabajo hemos intentado encajar el papel de las analogías dentro de un esquema de evolución conceptual más general como el que propone Clement (2000). Además, hemos incorporado a dicho esquema la distinción que introducen Galagovsky y Adúriz-Bravo (2001) al hablar de modelos científicos y representaciones didácticas de esos modelos, así como la distinción entre modelos mentales y conceptuales (Greca y Moreira, 2000) y la noción de modelo subyacente a la analogía que sugiere Duit (1991).

Dicho esquema puede considerarse la base para el desarrollo de un modelo de intervención mediante analogías, que fue gestándose paralelamente al desarrollo de esta investigación (Oliva et al., 2001) y que ha impregnado parcialmente el diseño didáctico que hemos utilizado en la parte experimental de este trabajo. Su objetivo era evaluar la incidencia del uso de analogías en la construcción de un modelo cinéntico-molecular por parte de alumnos de secundaria obligatoria.

Refiriéndonos ya a los resultados obtenidos, diremos que el uso de analogías, como recurso didáctico, parece ser de utilidad a la hora de facilitar la construcción en los alumnos de ideas asociadas al modelo cinético-molecular. Aquellos alumnos que fueron enseñados mediante analogías alcanzaron finalmente una mayor facilidad para evocar modelos explicativos próximos al modelo objeto que se planteó como meta del aprendizaje, que aquellos otros que fueron enseñados sin ellas. Si bien dicho efecto fue limitado y no pudo detectarse en todas y cada una de las tareas, sí al menos pudo apreciarse en aquéllas que mostraban un mayor nivel de complejidad. Así, pues, los resultados obtenidos parecen sostener la hipótesis de que las analogías pueden jugar un papel favorable en el desarrollo de la visión de los alumnos sobre un modelo de la materia; y aportan, además, un cierto soporte favorable a la forma de utilizar analogías según las propuestas que hemos desarrollado en éste y anteriores estudios.

Una forma de explicar las discretas diferencias encontradas entre el grupo experimental y el de control, significativas pero inferiores a las esperadas, podría ser teniendo en cuenta la superioridad en el estatus académico del que partieron los alumnos de este último grupo con respecto a los del primero. Otra posible interpretación se basaría en las grandes similitudes metodológicas que afectaban a ambos grupos, factor que, tal vez, habría llegado a diluir cualquier efecto diferenciador que el uso de analogías hubiera podido introducir. Por último, es factible, asimismo, que la utilización de analogías durante la intervención didáctica no siempre garantizase la comprensión de las mismas en los límites pretendidos. Si ello es así, es previsible que las analogías no siempre lleguen a jugar el papel esperado, al menos en aquellos casos en los que éstas no son comprendidas o no son interpretadas en los términos esperados.

En resumidas cuentas, los resultados obtenidos pueden considerarse prometedores y nos animan a seguir investigando sobre el tema. Concretamente, en un trabajo que tenemos en preparación analizamos aspectos tales como el grado de comprensión de los alumnos sobre las analogías utilizadas en clase y cómo dicha comprensión afectaba al nivel de adecuación de los modelos evocados sobre la estructura interna de la materia. También queda pendiente el análisis de las actitudes que presentan los alumnos acerca de las analogías como recurso educativo, capítulo que también reservamos para dicho trabajo.

\section{NOTA}

* Una primera versión de este trabajo se presentó como comunicación en el VI Congreso de Investigación en la Didáctica de las Ciencias (Barcelona, septiembre de 2001). 


\section{REFERENCIAS BIBLIOGRÁFICAS}

ARAGÓN, M.M., BONAT, M., OLIVA, J.M. y MATEO, J. (1999). Las analogías como recurso didáctico en la enseñanza de las ciencias. Alambique, 22, pp. 109-116.

AAVV (1994). El conocimiento escolar. Investigación en la Escuela, 23.

BENARROCH, A. (2000). El desarrollo cognoscitivo de los estudiantes en el área de la naturaleza corpuscular de la materia. Enseñanza de las Ciencias, 18(2), pp. 235-246.

BENARROCH, A. (2001). Una interpretación del desarrollo cognoscitivo de los alumnos en el área de la naturaleza corpuscular de la materia. Enseñanza de las Ciencias, 19(1), pp. 123-134.

CLEMENT, J.J. (2000). Model based learning as a key research area for science education. International Journal of Science Education, 22(9), pp. 1041-1053.

DAGHER, Z.R. (1994). Does the use of analogies contribute to conceptual change? Science Education, 78(6), pp. 601-614.

DAGHER, Z.R. (1995). Review of studies on the effectiveness of instructional analogies in science education. Science Education, 79(3), pp. 295-312.

DREYFUS, A., JUNGWIRTH, E. y ELIOVITCH, R. (1990). A applying the «cognitive conflict» strategy for conceptual change, some implications, difficulties and problems. Science Education, 74(5), pp. 555-569.

DUIT, R. (1991). On the role of analogies and metaphors in learning science. Science Education, 75(6), pp. 649-672.

DRIEL, J.H. VAN y VERLOOP, N. (1999). Teachers' knowledge and modelling in science. International Journal of Science Education, 21(11), pp. 1141-1153.

FURIÓ, C., BARRENETXEA, I. y REYES, J.V. (1994). Contribución de la resolución de problemas como investigación al paradigma constructivista de aprendizaje de las ciencias. Investigación en la Escuela, 24, pp. 88-99.

GALAGOVSKY, L. y ADÚRIZ-BRAVO, A. (2001). Modelos y analogías en la enseñanza de las ciencias naturales. El concepto de modelo didáctico analógico. Enseñanza de las Ciencias, 19(2), pp. 231-242.

GILBERT, J.K. (1993). Models and modelling in science education. Hatfield, UK: Association for Science Education (citado por Harrison y Tregust, 2000).

GILBERT, J.K., BOULTER, C. y RUTHERFORD, M. (1998). Models in explanations, Part 1: Horses for courses? International Journal of Science Education, 20(1), pp. 83-97.

GLYN, S.M. (1991). Explaining science concepts: A teaching with analogies model, en Glyn, S.M., Yeany, R.H. y Britton, B.K. (eds.), The psychology of learning science. Hillsdale, N.J.: Lawrence Erlbaum (citado por Dagher, 1995b).

GONZÁLEZ-LABRA, M.J. (1997). Aprendizaje por analogía: análisis del proceso de inferencia analógica para la adquisición de nuevos conocimientos. Madrid: Trotta.

GRECA, I.M. y MOREIRA, M.A. (1998). Modelos mentales y aprendizaje de física en electricidad y magnetismo. Enseñanza de las Ciencias, 16(2), pp. 289-304.
GRECA, I.M. y MOREIRA, M.A. (2000). Mental models, conceptual models and modelling. International Journal of Science Education, 22(1), pp. 1-11.

GROSSLIGHT, L., UNGER, C., JAY, E. y SMITH, C. (1991). Understanding models and their use in science conceptions of middle and high school teachers and experts. Journal of Research in Science Teaching, 28(9), pp. 799-882.

GUTIÉRREZ, R. (1996). Modelos mentales y concepciones espontáneas. Alambique, 7, pp. 73-87.

GUTIÉRREZ, R. y OGBORN, J. (1992). A causal framework for analysing alternative conceptions. International Journal of Science Education, 14(2), pp. 201-220.

HALLOUN, I. (1996). Schematic modelling for meaningful learning in physics. Journal of Research in Science Teaching, 33(9), pp. 1019-1041.

HARRISON, A.G. y TREAGUST, D.F. (2000a). A tipology of school science models. International Journal of Science Education, 22(9), pp. 1011-1026.

HARRISON, A.G. y TREAGUST, D.F. (2000b). Learning about atoms. Molecules and chemical bonds: a case study of multiple model use in grade 11 chemistry. Science Education, 84, pp. 352-381.

HEWSON, P. y HEWSON, M. (1992). The status of student conceptions, en Duit, R., Golberg, F. y Niedderer, H. (eds.). Research in physics learning: Theoretical issues and empirical studies. Kiel (Alemania): Institute of Science Education.

HEYWOOD, D. y PARKER, J. (1997). Confronting the analogy: primary teachers exploring the usefulness: in the teaching and learning of electricity. International Journal of Science Education, 19(8), pp. 869-885.

HOLLAND, J.H., HOLYOAK, K.J., NISBETT, R.E. y THAGARD, P.R. (1986). Induction: processes of inference, learning and discovery. Cambridge, Mass: The MIT Press.

JUSTI, R. y GILBERT, J. (2000). History and philosophy of science through models: some chellenges in the case of «the atom». International Journal of Science Education, 22(9), pp. 993-1009.

JOHNSON-LAIRD, P.N. (1983). Mental models. Cambridge: Cambridge - University Press.

LINDER, C.J. (1993). A challenge to conceptual change. Science Education, 77(3), pp. 293-300.

MALONEY, D.P. y SIEGLER, R.S. (1993). Conceptual competition in physics learning. International Journal of Science Education, 15(3), pp. 283-295.

MARÍN, N. (2003). Conocimientos que interaccionan en la enseñanza de las ciencias. Enseñanza de las Ciencias, 21(1), pp. 65-78.

OLIVA, J.M. (1999). Algunas reflexiones sobre las concepciones alternativas y el cambio conceptual. Enseñanza de las Ciencias, 17(1), pp. 93-108.

OLIVA, J.M e IGLESIAS, A. (1989). La interacción entre 
estudiantes en el trabajo en grupos y la construcción del modelo corpuscular de la materia y el principio de conservación de la masa. Investigación en la Escuela, 12, pp. 57-67.

OLIVA, J.M, ARAGÓN, M.M., MATEO, J. y BONAT, M. (2001). Una propuesta didáctica, basada en la investigación, para el uso de analogías en la enseñanza de las ciencias. Enseñanza de las Ciencias, 19(3), pp. 453-470.

POZO, J.I. (1989). Teorías cognitivas del aprendizaje. Madrid: Morata.

POZO, J.I., GÓMEZ CRESPO, M.A., LIMÓN, M. y SANZ, A. (1991). Procesos cognitivos en la comprensión de la ciencia: las ideas de los adolescentes sobre la química. Madrid: CIDE.

REINER, M. y GILBERT, J. (2000). Epistemological resources for thought experimentation in science education. International Journal of Science Education, 22(5), pp. 489-506.

SÁNCHEZ-BLANCO, G. y VALCÁRCEL, M.V. (1993). Diseño de unidades didácticas en el área de ciencias experimentales. Enseñanza de las Ciencias, 11(1), pp. 33-44.
SÁNCHEZ-BLANCO, G., PRO, A. y VALCÁRCEL, M.V. (1997). La utilización de un modelo de planificación de unidades didácticas: el estudio de las disoluciones en la educación secundaria. Enseñanza de las Ciencias, 15(1), pp. 35-50.

SOLSONA, N., IZQUIERDO, M. y GUTIÉRREZ, R. (2000). El uso de razonamientos causales en relación con la significatividad de los modelos teóricos. Enseñanza de las Ciencias, 18(1), pp. 15-23.

TABER, K.S. (2001). Shifting sands: a case study of conceptual development as competition between alternative conceptions. International Journal of Science Education, 23(7), pp. 731-753.

THAGARD, P. (1992). Analogy, explanation and education. Journal of Research in Science Teaching, 29, pp. 537-544.

VOSNIADOU, S. (1994). Capturing and modelling the process of conceptual change. Learning and Instruction, 4(1), pp. 45-69. 


\section{ANEXO I}

1) Observa un bote de lentejas y una red. Compara las visiones de los dos sistemas según sea de cerca o de lejos.

¿Serían comparables las lentejas al conjunto de moléculas que constituyen las sustancias puras? ¿Puede compararse la red con la estructura de los sólidos? Indica las semejanzas $\mathrm{y}$ diferencias.
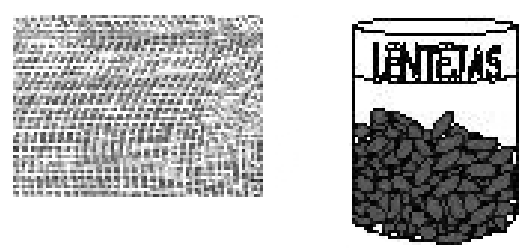

2 a) Imaginemos la clase como un recipiente y cada uno de vosotros como una molécula, ¿cómo representarías los estados de la materia?

2 b) ¿Qué diferencias existen entre vuestra representación y la constitución real de la materia?

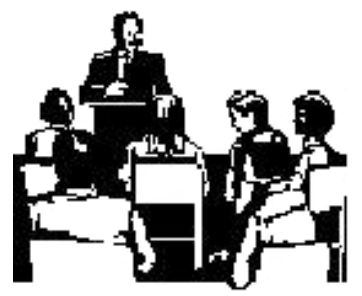

\section{ANEXO II}

Tarea 3. Tenemos dos recipientes iguales. El recipiente A está lleno de agua liquida y el B de vapor de agua. Mediante la balanza observamos que el recipiente que contiene agua líquida (A) es más pesado que el que contiene vapor (B). ¿Cómo crees que es posible que volúmenes iguales de la misma sustancia tengan peso diferente?

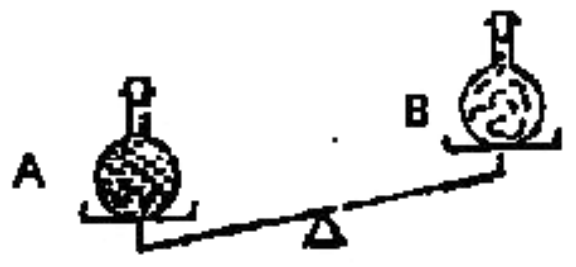

